\title{
Neutron properties from light nuclei
}

\author{
Evgeny Epelbaum ${ }^{1, \star}$, Hans-Werner Hammer ${ }^{2,3, \star \star}$ and Ulf-G. Meißner ${ }^{4,5, \star \star \star}$ \\ ${ }^{1}$ Institut für Theoretische Physik II, Ruhr-Universität Bochum, D-44780 Bochum, Germany \\ ${ }^{2}$ Institut für Kernphysik, Technische Universität Darmstadt, 64289 Darmstadt, Germany \\ ${ }^{3}$ ExtreMe Matter Institute EMMI, GSI Helmholtzzentrum für Schwerionenforschung GmbH, 64291 Darmstadt, Germany \\ ${ }^{4}$ Universität Bonn, Helmholtz Institut für Strahlen- und Kernphysik, Bethe Center for Theoretical Physics, D-53115 Bonn, Germany \\ ${ }^{5}$ Forschungszentrum Jülich, Institut für Kernphysik (IKP-3), Institute for Advanced Simulation (IAS-4) and Jülich Center for Hadron \\ Physics, D-52145 Jülich, Germany
}

\begin{abstract}
We review the achievements of the project B.6. Topics addressed include pion photoproduction of three-nucleon systems, electromagnetic and axial currents in chiral nuclear EFT, aspects of EFTs with spin$3 / 2$ fields, nuclear structure and dynamics from the pionless EFT, the development and applications of highprecision two-nucleon forces and pion production in nucleon-nucleon collisions.
\end{abstract}

\section{Introduction}

The project B.6 has been funded over the second and third period of the SFB/TR 16. It grew out of the project $\mathbf{B . 3}$ from the first funding period, as explained in the corresponding contribution to that project. In the second funding period, the PIs were Evgeny Epelbaum and HansWerner Hammer. After about one year in the third funding period, Hans-Werner Hammer moved to Darmstadt and was replaced as PI by Ulf Meißner, however, the collaboration continued. The project focused on few-nucleon physics in various settings of nuclear EFT (pionless and pionfull) as well as on electroweak currents in nuclei, pion production in nucleon-nucleon collisions and formal aspects related to gauge invariance and the $\Delta(1232)$ resonance.

Our contribution is organized as follows: Section 2 summarizes the results on pion photoproduction off threenucleon systems. In Sec. 3, we display the results obtained for the electromagnetic currents in chiral nuclear EFT and a first application to deuteron disintegration. Sec. 4 is devoted to some formal developments of including spin-3/2 particles in chiral EFTs. In Sec. 5, the applications of the so-called pionless EFT to nucleon-deuteron scattering, the halo nucleus ${ }^{11} \mathrm{Be}$ and finite volume methods in the threeparticle system are discussed. A novel approach to the two-nucleon system based on a coordinate-space representation and a novel method for error quantification are described in Sec. 6. The rather involved calculation to generate consistent axial currents is briefly summarized in Sec. 7 and aspects of pion production in nucleon-nucleon collisions are displayed in Sec. 8. Finally, an important

\footnotetext{
$\star$ e-mail: evgeny.epelbaum @ rub.de

$\star \star$ e-mail: hammer@ theorie.ikp.physik.tu-darmstadt.de

$\star \star \star$ e-mail: meissner@hiskp.uni-bonn.de
}

disclaimer: This is the review of the project B.6 but not a general review of the various fields to which this research contributed. Therefore, only the papers that resulted from this research are quoted, with the exception of a few external papers and of course, in case data were involved, with proper reference to the experimental papers. The links to the other projects are mentioned in passing, but no effort is made to discuss these in any detail.

\section{Pion photoproduction off three-nucleon systems}

Threshold neutral pion photo- and electroproduction off the nucleon is one of the finest reactions to test the chiral QCD dynamics. While elementary proton targets are accessible directly in experiment, pion production off neutrons requires the use of nuclear targets like the deuteron or three-nucleon bound states like ${ }^{3} \mathrm{H}$ (triton) or ${ }^{3} \mathrm{He}$. Of particular interest is to test the counterintuitive chiral perturbation theory (CHPT) prediction that the elementary neutron S-wave multipole $E_{0+}^{\pi^{0} n}$ is larger in magnitude than the corresponding one of the proton, $E_{0+}^{\pi^{0} p}[1,2]$. This prediction was already successfully tested in neutral pion photo[3] and electroproduction off the deuteron [4]. However, given the scarcity and precision of the corresponding data, it is mandatory to study also pion production off threenucleon bound states, that can be calculated nowadays to high precision based on chiral nuclear effective field theory (EFT), that extends CHPT to nuclear physics, for a recent detailed review, see Ref. [5]. Note that ${ }^{3} \mathrm{He}$ appears to be a particularly promising target to extract the information about the neutron amplitude. Its wave function is strongly dominated by the principal "s-state" component which suggests that the spin of ${ }^{3} \mathrm{He}$ is largely driven by the one of the neutron. 
In Ref. [6], we have presented a calculation of neutral pion production off ${ }^{3} \mathrm{H}$ and ${ }^{3} \mathrm{He}$ at threshold to leading oneloop order for the production operator in the framework of chiral nuclear effective field theory. We used the chiral wave functions from Epelbaum, Glöckle and Meißner, which are consistent with the pion production operator to calculate the $\mathrm{S}$-wave $3 \mathrm{~N}$ multipoles $E_{0+}$ and $L_{0+}$. To this order, the production operator gets both one- and twobody contributions. Our calculation shows that the twobody contributions are of the same order of magnitude as the one-body contributions. A similar behavior was observed in the deuteron case [3]. The theoretical uncertainty resulting from the cutoff variation in the employed wave functions appears to be small (of the order of $3 \%$ ). The dominant theoretical error at this order stems from the threshold pion production amplitude off the proton and the neutron, which is estimated to be about $5 \%$. We explored the sensitivity of neutral pion photoproduction on ${ }^{3} \mathrm{He}$ to the elementary neutron multipole $E_{0+}^{\pi^{0} n}$ and found a large sensitivity. This makes ${ }^{3} \mathrm{He}$ a promising target to test the counterintuitive CHPT prediction for $E_{0+}^{\pi^{0} n}[1,2]$. The cutoff variation estimate leads to a very small error for the $2 \mathrm{~N}$ contribution. If the error of this contribution is artificially enlarged by a factor of 10 , the extraction of the neutron multipole is still feasible experimentally. We have shown that our prediction for the ${ }^{3} \mathrm{He} \mathrm{S}$-wave multipole $E_{0+}$ is roughly consistent with the value deduced from the old Saclay measurement of the threshold cross section [7]. A new measurement using modern technology and better methods to deal with few-body dynamics is urgently called for.

In Ref. [8], we have extended this calculation to fourth order in the chiral expansion, including consistently all next-to-next-to-leading order contributions in the standard heavy baryon expansion. This amounts to a complete (i.e. subleading) one-loop calculation in the one-nucleon sector. Apart from the fourth order corrections already included in the single nucleon multipoles $E_{0+}$ and $L_{0+}$, there are various fourth order corrections that arise due to the presence of the other two nucleons in the tri-nucleon system considered here. First, there are the boost corrections that arise from the observation that within a nucleus the threshold for pion production is shifted compared to the free threshold. For light systems as considered here, this essentially induces P-wave contributions. Second, there are further corrections to the two-nucleon production operator, that can be grouped in two categories, namely the socalled static and recoil corrections. The static corrections involve - as the leading $2 \mathrm{~N}$ corrections do - static propagators but one insertion from the dimension two chiral effective pion-nucleon Lagrangian. The recoil contributions feature corrections to the static propagators with only insertions from the leading order (dimension one) chiral Lagrangian. With the fourth-order single nucleon input from heavy baryon CHPT $[1,2,9]$ (the latter result obtained in project B.3), we obtain for the threshold multipoles on ${ }^{3} \mathrm{He}$ and on ${ }^{3} \mathrm{H}$ the values listed in Table 1 . We find that the fourth order corrections for the electric dipole amplitude $E_{0+}$ for both tri-nuclear systems come out to be very small,

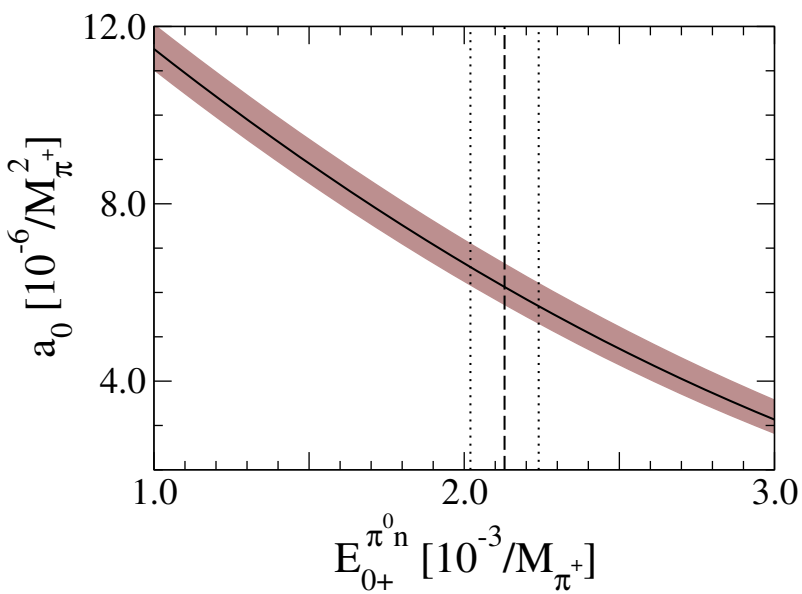

Figure 1. Sensitivity of the S-wave cross section $a_{0}$ for ${ }^{3} \mathrm{He}$ in units of $10^{-6} / M_{\pi^{+}}^{2}$ to the single-neutron multipole $E_{0+}^{\pi^{0} n}$ in units of $10^{-3} / M_{\pi^{+}}$. The vertical dashed line gives the CHPT prediction for $E_{0+}^{\pi^{0} n}$ and the vertical dotted lines indicate the 5\% error in the prediction. The shaded band indicates the theory error estimated from the cutoff variation and a $5 \%$ error in $E_{0+}^{\pi^{0} p}$.

much smaller than in case of the deuteron. In Fig. 1, we illustrate the sensitivity of the S-wave cross section $a_{0}$ to the single-neutron multipole $E_{0+}^{\pi^{0} n}$. The shaded band indicates the theory error estimated from the cutoff variation as described above and a $5 \%$ error in $E_{0+}^{\pi^{0} p}$. As shown above, the uncertainties related to the nuclear effects are of the order of one percent, i.e. completely negligible. So our estimate of a $10 \%$ uncertainty of the $2 \mathrm{~N}$ contributions in Ref. [6] driven by the analogy to the deuteron case [3] turns out to be much too conservative.

\section{Electromagnetic currents in chiral nuclear EFT}

One of the main advantages of chiral nuclear EFT is the possibility of deriving a set of consistent forces and electroweak currents. This connection was first explored for the electromagnetic currents. A powerful tool to perform such consistent calculations is the so-called method of unitary transformations, that had been used earlier by the Bochum group in the context of the meson-theoretical description of nuclear forces and currents.

In Ref. [10], we calculated the leading two-pion exchange two-nucleon four-current operator in chiral EFT based on the method of unitary transformation which we used to derive nuclear forces in Refs. [11-15], see the pertinent diagrams in Fig. 2. Our work provides an important check of the results presented in Refs. [16, 17] but also differs from these works in several important respects. First, we use a completely different method to compute the current operator. Secondly, we also give results for the exchange charge density which, to the best of our knowledge, have not yet been published before. Finally, we evaluate analytically all loop integrals to obtain a representation in momentum space in terms of the standard loop functions and the three-point functions. The latter are reduced to a form which can be easily treated numerically. We also 
Table 1. Numerical results for the $3 \mathrm{~N}$ multipoles. The first error is our estimation of the theoretical uncertainty resulting from the truncation of the chiral expansion while the second one is the statistical error from the Monte Carlo integration, see Ref. [8] for more details. For the total result only the combined error is given.

\begin{tabular}{|c||c|c|c|c|c|c|}
\hline${ }^{3} \mathrm{He}$ & $1 \mathrm{~N}\left(q^{4}\right)$ & $2 \mathrm{~N}\left(q^{3}\right)$ & $1 \mathrm{~N}-$ boost & $2 \mathrm{~N}$-static $\left(q^{4}\right)$ & $2 \mathrm{~N}-$ recoil $\left(q^{4}\right)$ & total \\
\hline$E_{0+}\left[10^{-3} / M_{\pi^{+}}\right]$ & $+1.71(4)(9)$ & $-3.95(3)$ & $-0.23(1)$ & $-0.02(0)(1)$ & $+0.01(2)(1)$ & $-2.48(11)$ \\
$L_{0+}\left[10^{-3} / M_{\pi^{+}}\right]$ & $-1.89(4)(9)$ & $-3.09(2)$ & $-0.00(0)$ & $-0.07(1)(1)$ & $+0.07(7)(0)$ & $-4.98(12)$ \\
\hline${ }^{3} \mathrm{H}$ & $1 \mathrm{~N}\left(q^{4}\right)$ & $2 \mathrm{~N}\left(q^{3}\right)$ & $1 \mathrm{~N}-$ boost & $2 \mathrm{~N}$-static $\left(q^{4}\right)$ & $2 \mathrm{~N}-$ recoil $\left(q^{4}\right)$ & total \\
\hline$E_{0+}\left[10^{-3} / M_{\pi^{+}}\right]$ & $-0.93(3)(5)$ & $-4.01(3)$ & $-0.35(1)$ & $-0.02(1)(1)$ & $+0.01(2)(0)$ & $-5.28(7)$ \\
$L_{0+}\left[10^{-3} / M_{\pi^{+}}\right]$ & $-0.99(4)(5)$ & $-3.13(1)$ & $-0.02(0)$ & $-0.07(0)(1)$ & $+0.07(7)(0)$ & $-4.14(10)$ \\
\hline
\end{tabular}

Class 1:

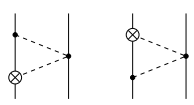

Class 2:

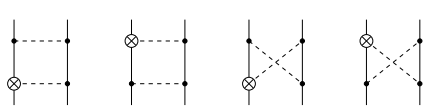

Class 3:

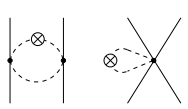

Class 4:
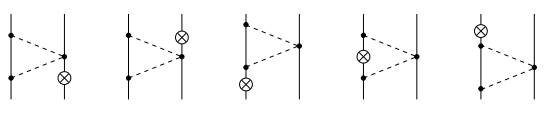

Class 5:<smiles>CC(C)(C)C1(C)C(I)C1(C)C</smiles>

Class 6:
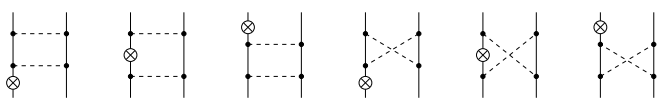

Class 7:
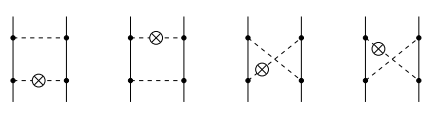

Figure 2. Diagrams showing contributions to the leading twopion exchange currents. Solid and dashed lines refer to nucleons and pions, respectively. Solid dots are the lowest-order vertices from the effective Lagrangian while the crosses represent insertions of the electromagnetic vertices. Diagrams resulting from interchanging the nucleon lines are not shown.

succeeded to analytically carry out the Fourier transformation for all contributions leading to an extremely compact representation of the current and charge densities in coordinate space.

In Ref. [18], we applied the method of unitary transformation to work out the leading loop contributions to the one-pion exchange and short-range two-nucleon electromagnetic current and charge densities. The renormalized expressions for the one-pion exchange charge and current operators are given for the first time. We have discussed in detail renormalization of the one-pion exchange contributions which provides a stringent test of our theoretical approach. More precisely, all emerging ultraviolet divergences have to be absorbed into redefinition of the lowenergy constants $l_{i}$ and $d_{i}$ entering the Lagrangians $\mathcal{L}_{\pi \pi}^{(4)}$ and $\mathcal{L}_{\pi N}^{(3)}$, respectively. There is no freedom in this procedure as the corresponding $\beta$-functions of all these LECs in dimensional regularization are fixed and well known. We have demonstrated that it is indeed possible to renormalize the one-loop contributions provided one makes use of the freedom to employ additional unitary transformations. Further, we succeeded to obtain compact, analytical expressions for the current and charge densities both in momentum and coordinate spaces which can be used in future numerical calculations. Finally, we provide a detailed comparison between our results and the ones obtained by Pastore et al. [19] within a different framework, and we spelled out the deficiencies of that calculation.

The long-range two-pion exchange (TPE) contributions to the nuclear current operator which appear at nextto-leading order (NLO) of the chiral expansion were used to describe electromagnetic processes in Ref. [20]. Their role in the photodisintegration of ${ }^{2} \mathrm{H}$ and ${ }^{3} \mathrm{He}$ was studied and the corresponding predictions were compared with experimental data. The bound and scattering states are calculated using five different parametrizations of the chiral next-to-next-to-leading order nucleon-nucleon potential which allows to estimate the theoretical uncertainty at a given order in the chiral expansion. For some observables the results are very close to the predictions based on the AV18 NN potential. In the most cases, the addition of the long-range TPE currents improved the description of the experimental data. Last but not least, in Ref. [21] we have used the novel contributions to the exchange current operator to analyze the magnetic form factors of the deuteron.

\section{Formal aspects of spin-3/2 fields}

In the paper [22], we have explicitly constructed a nonlinear field transformation that brings the interaction Lagrangian of spin-3/2 fields as given by

$$
\mathcal{L}=\bar{\psi}(i \partial-m+V) \psi,
$$

in a form that is invariant under gauge transformation,

$$
\psi_{\mu} \rightarrow \psi_{\mu}+\partial_{\mu} \epsilon
$$

This proves a conjecture made in Ref. [23] where it was shown how a gauge non-invariant Lagrangian with linear couplings in the spin-3/2 fields can be brought into a gauge-invariant form via a suitably chosen field redefinition. We have also given a diagrammatic explanation of our main result, which allows to one split the truly propagating spin-3/2 components from the non-propagating 


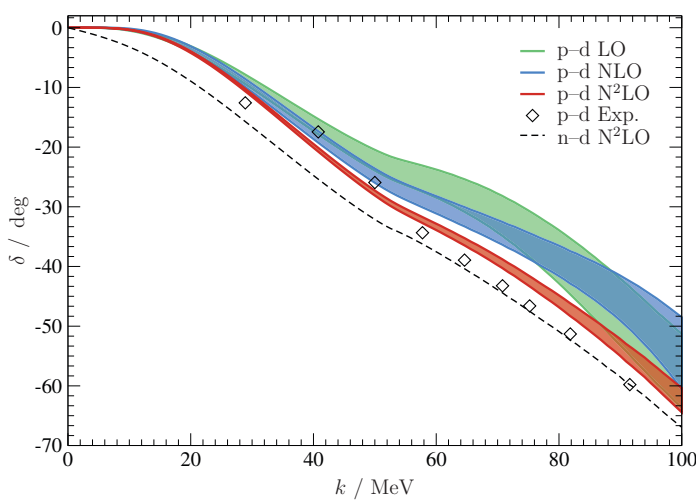

Figure 3. $N-d$ doublet channel S-wave scattering phase shifts as functions of the center-of-mass momentum $k$ at various orders in the EFT expansion compared to the data. Error bands are generated by cutoff variation from 200 to $600 \mathrm{MeV}$.

spin-1/2 contributions that are subsumed in local operators existing in the most general Lagrangian of deltas coupled to nucleons, pions and external fields.

In Ref. [24], we have further proven to all orders in the small scale expansion that all off-shell parameters which appear in the chiral effective Lagrangian with explicit $\Delta$ (1232)-isobar degrees of freedom can be absorbed into redefinitions of certain low-energy constants and are therefore redundant.

\section{Nuclear structure and dynamics from the pionless EFT}

At energies well below the pion mass, one can describe nuclear interactions and dynamics based on a non-relativistic Lagrangian with nucleon contact interactions only, the so-called pionless EFT. In Ref. [25], low-energy protondeuteron scattering was calculated in this framework. In the quartet channel, the elastic scattering phase shift was worked out up to next-to-next-to-leading order in the power counting. In the doublet channel, a next-to-leading order calculation was performed. We obtained good agreement with the available phase shift analyses down to the scattering threshold. The phase shifts in the region of the non-perturbative Coulomb interactions were calculated by using an optimized integration mesh. Moreover, the Coulomb contribution to the ${ }^{3} \mathrm{He}-{ }^{3} \mathrm{H}$ binding energy difference was evaluated in first order perturbation theory. In Ref. [26], we have computed $E 1$ transitions and electric radii in the Beryllium-11 nucleus using an EFT that exploits the separation of scales in this halo system. We fix the leading-order parameters of the EFT from measured data on the $\frac{1}{2}^{+}$and $\frac{1}{2}^{-}$levels in Be-11 and the $B(E 1)$ strength for the transition between them. We then obtain predictions for the $B(E 1)$ strength for Coulomb dissociation of the Be-11 nucleus to the continuum. We also compute the charge radii of the $\frac{1}{2}^{+}$and $\frac{1}{2}^{-}$states. Agreement with experiment within the expected accuracy of a leading-order computation in this EFT is obtained. We also discuss how next-to-leading-order (NLO) corrections involving both s-wave and p-wave neutron-Be-10 interactions affect our results, and display the NLO predictions

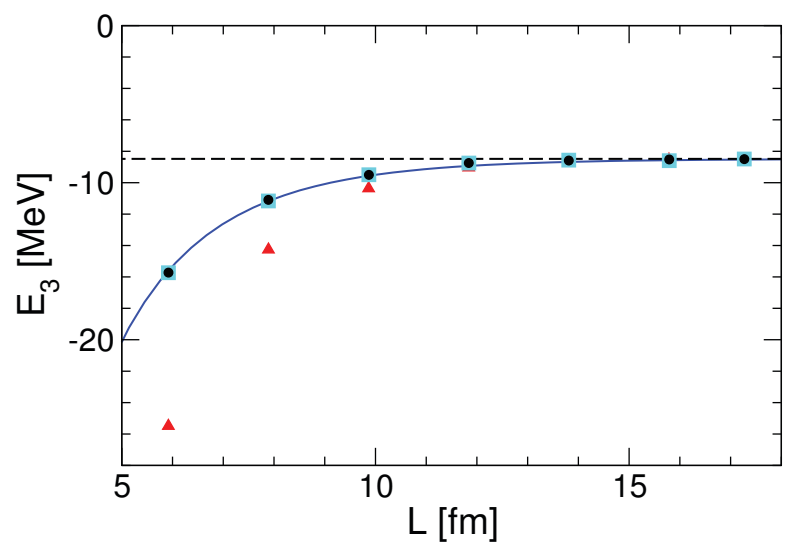

Figure 4. Triton energy $E_{3}$ for different edge lengths $L$ of the cubic volume. Squares and circles are data sets for different cutoffs. The triangles are from a nuclear lattice calculation using chiral EFT at $\mathrm{N}^{2} \mathrm{LO}$ [31]. The horizontal dashed line indicates the physical triton binding energy, while the solid line is a fit to our results.

for quantities which are free of additional short-distance operators at this order. Information on neutron-Be-10 scattering in the relevant channels is inferred.

Another interesting set of studies, that is closely linked to the finite volume studies of baryon resonances in project A.6 was published in Refs. [27-29]. The starting point is the observation that three bosons with large scattering length show universal properties that do not depend on the details of the interaction at short distances. In the threeboson system, these properties include a geometric spectrum of shallow three-body states called "Efimov states" and log-periodic dependence of scattering observables on the scattering length. In Ref. [27] the modification of the Efimov states in a finite cubic box was analyzed and the dependence of their energies on the box size was calculated using effective field theory. The renormalization of the effective field theory in the finite volume was also explicitly verified. These studies were extended in Ref. [28]. Previous calculations for positive scattering length that were perturbative in the finite volume energy shift are extended to arbitrarily large shifts and negative scattering lengths. The renormalization of the effective field theory in the finite volume is explicitly verified. Moreover, we investigate the effects of partial wave mixing and study the behavior of shallow trimers near the dimer energy. Further, we provide numerical evidence for universal scaling of the finite volume corrections. Finally, understanding the volume dependence of the triton binding energy is an important step towards lattice simulations of light nuclei. In Ref. [29] the triton binding energy in a finite cubic box with periodic boundary conditions to leading order in the pionless EFT was calculated. Higher order corrections are estimated and the proper renormalization of our results is verified explicitly. We present results for the physical triton, see Fig. 4, as well as for the pion-mass dependence of the triton spectrum near the "critical" pion mass, $M_{\pi}^{c} \sim 197 \mathrm{MeV}$, where chiral effective field theory suggests that the nucleon-nucleon scattering lengths in the singlet- and triplet-channels may diverge simultaneously. 
An extension of the Lüscher formula to the three-body system is implicit in these results. Such an explicit formula was supplied only much later in Ref. [30].

\section{High-precision NN forces: Foundations and applications}

In the paper [32], we have presented a new generation of $\mathrm{NN}$ potentials derived in chiral EFT up to $\mathrm{N}^{3} \mathrm{LO}$. The new chiral forces offer a number of substantial improvements as compared to the widely used $\mathrm{N}^{3} \mathrm{LO}$ potentials of Refs. [33, 34] introduced a decade ago. First of all, we employ a local regularization scheme for the pion exchange contributions which, differently to the standard nonlocal regularization applied e.g. in Refs. [33, 34], does not distort the low-energy analytic structure of the amplitude and, as a consequence, leads to a better description of phase shifts and experimental data. The employed regulator, by construction, removes the short-range part of the chiral two-pion exchange and thus makes the additional spectral function regularization used in the potential of Ref. [33] obsolete. This is a particularly welcome feature given that the expressions for the three-nucleon force at $\mathrm{N}^{3} \mathrm{LO}$ and $\mathrm{N}^{4} \mathrm{LO}$ are only available in the framework of dimensional regularization. Further, in contrast to the earlier studies of Refs. [33, 34], we have taken all pion-nucleon LECs and especially the subleading LECs $c_{i}$ from pion-nucleon scattering without any fine tuning. The LECs accompanying NN contact interactions were determined by fits to the Nijmegen phase shifts and mixing angles for five different values of the coordinate-space cutoff $R$ chosen in the range of $R=0.8 \ldots 1.2 \mathrm{fm}$ and appear to be of natural size in all cases. The new $\mathrm{N}^{3} \mathrm{LO}$ potentials allow for an excellent description of the Nijmegen np and pp phase shifts at energies below $200 \mathrm{MeV}$ and, for the cutoff choices of $R=0.9 \mathrm{fm}$ and $R=1.0 \mathrm{fm}$, even up to $E_{\text {lab }}=300 \mathrm{MeV}$. Furthermore, the deuteron properties are accurately described. Moreover, the deuteron wave functions are free from distortions at distances larger than $r \sim 2 \ldots 3 \mathrm{fm}$ which appear for the $\mathrm{N}^{3} \mathrm{LO}$ potentials of Refs. [33, 34] due to the employed form of the regulator. We found that the description of the Nijmegen phase shifts improves substantially when going from LO to NLO, from NLO to $\mathrm{N}^{2} \mathrm{LO}$ and from $\mathrm{N}^{2} \mathrm{LO}$ to $\mathrm{N}^{3} \mathrm{LO}$ as one expects for a convergent expansion. It is worth to emphasize in this connection that the short range part of the NLO and $\mathrm{N}^{2} \mathrm{LO}$ potentials involves the same set of operators. Our findings therefore provide yet another evidence of the subleading two-pion exchange which was also observed in earlier studies. As an important consistency check of our approach, we have studied the residual cutoff dependence of phase shifts at different orders in the chiral expansion. We found, in particular, that the cutoff dependence is strongly reduced at $\mathrm{N}^{3} \mathrm{LO}$ compared to $\mathrm{N}^{2} \mathrm{LO}$ in the whole considered range of energies. To illustrate these features, we consider the chiral expansion of the np total cross section at the energies of $E_{\text {lab }}=50,96,143$ and $200 \mathrm{MeV}$ based on the interactions introduced in the previous sections as shown in Fig. 5
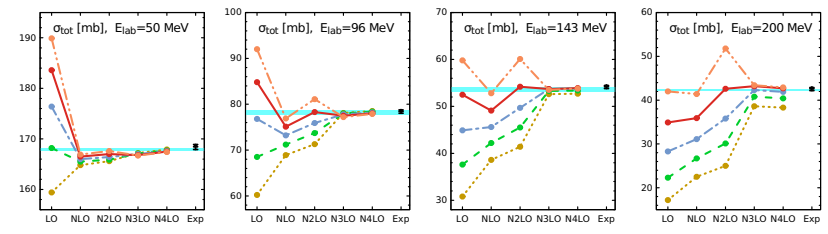

Figure 5. Order-by-order convergence of the chiral expansion for the np total cross section at energies of $E_{\text {lab }}=50 \mathrm{MeV}$, $E_{\text {lab }}=96 \mathrm{MeV}$ and $E_{\text {lab }}=143 \mathrm{MeV}$ and $E_{\text {lab }}=200 \mathrm{MeV}$. Dotted (light brown), dashed (green), dashed-dotted (blue), solid (red) and dashed-double-dotted (pink) lines show the results based on the cutoff $R=1.2 \mathrm{fm}, R=1.1 \mathrm{fm}, R=1.0 \mathrm{fm}, R=0.9 \mathrm{fm}$ and $R=0.8 \mathrm{fm}$, respectively. The horizontal band refers to the result of the Nijmegen PWA with the uncertainty estimated by means of deviations from the results based on the Nijmegen I, II and Reid 93 potentials as explained in the text. Also shown are experimental data of Ref. [35].

We have also addressed the issue of the uncertainty quantification of our results due to the truncation of the chiral expansion at a given order. In particular, we have argued that the standard procedure for error estimation based on a cutoff variation is not reliable and employed a simple alternative approach by directly estimating the expected size of higher-order contributions at a given energy. Such a procedure has the advantage of being applicable for any fixed value of the cutoff so that calculations based on different cutoffs can be used to provide additional consistency checks. Furthermore, disentangling the error analysis from the cutoff variation allows one to avoid an unnecessary increase of uncertainty due to softening the interaction. Notice that the versions of the potential corresponding to soft choices of the cutoff $R$ may still be useful for certain kinds of applications including, in particular, many-body calculations. We have applied this approach to the total np cross section at several energies and have verified that the results at different chiral orders and for different values of the cutoff are indeed consistent with each other. We have furthermore used this method to quantify the theoretical uncertainty in the description of the np phase shifts as well as differential cross sections and selected polarization observables in np scattering. In particular, we found the $\mathrm{N}^{3} \mathrm{LO}$ results for np scattering to be very accurate at energies below $\sim 100 \mathrm{MeV}$ with the corresponding error bands being barely visible and still rather accurate at the energy of $E_{\text {lab }}=200 \mathrm{MeV}$. In all considered cases, our results agree with the ones based on the Nijmegen PWA within the estimated theoretical accuracy. This gives us additional confidence in the reliability of the suggested way of quantifying the uncertainty. We have furthermore analyzed the uncertainties associated with making a specific choice of the functional form of the local regulator and employing the additional spectral function regularization of the TPEP and found them to be negligible at the level of the estimated theoretical accuracy at $\mathrm{N}^{3} \mathrm{LO}$.

In Ref. [36], we have presented a nucleon-nucleon potential at fifth order in chiral effective field theory. We find a substantial improvement in the description of nucleonnucleon phase shifts as compared to the fourth-order re- 
Table 2. $\chi^{2}$ /datum for the description of the Nijmegen np (upper two rows) and pp (lower two rows) phase shifts [37] at different orders in the chiral expansion for the cutoff $R=0.9 \mathrm{fm}$. Only those channels are included which have been used in the $\mathrm{N}^{3} \mathrm{LO} / \mathrm{N}^{4} \mathrm{LO}$ fits, namely the S-, $\mathrm{P}$ - and D-waves and the mixing angles $\epsilon_{1}$ and $\epsilon_{2}$.

\begin{tabular}{|cccccc|}
\hline$E_{\text {lab }}$ bin & LO & NLO & $\mathrm{N}^{2} \mathrm{LO}$ & $\mathrm{N}^{3} \mathrm{LO}$ & $\mathrm{N}^{4} \mathrm{LO}$ \\
\hline $0-100$ & 360 & 31 & 4.5 & 0.7 & 0.3 \\
$0-200$ & 480 & 63 & 21 & 0.7 & 0.3 \\
\hline $0-100$ & 5750 & 102 & 15 & 0.8 & 0.3 \\
$0-200$ & 9150 & 560 & 130 & 0.7 & 0.6 \\
\hline
\end{tabular}

sults of Ref. [32], see Tab. 2. This provides clear evidence of the corresponding two-pion exchange contributions with all low-energy constants being determined from pion-nucleon scattering. The fifth-order corrections to nucleon-nucleon observables appear to be of a natural size which confirms the good convergence of the chiral expansion for nuclear forces. Furthermore, the obtained results provide strong support for the novel way of quantifying the theoretical uncertainty due to the truncation of the chiral expansion proposed in Ref. [32], as shown for the np phase shifts in Fig. 6. This work opens up new perspectives for precision ab initio calculations in few- and many-nucleon systems and is especially relevant for ongoing efforts towards a quantitative understanding the structure of the three-nucleon force in the framework of chiral effective field theory.

In Ref. [39], we have applied these improved nucleonnucleon potentials up to fifth order in chiral EFT, along with a new analysis of the theoretical truncation errors, to study nucleon-deuteron $(\mathrm{Nd})$ scattering and selected lowenergy observables in ${ }^{3} \mathrm{H},{ }^{4} \mathrm{He}$, and ${ }^{6} \mathrm{Li}$. Calculations beyond second order differ from experiment well outside the range of quantified uncertainties, providing truly unambiguous evidence for missing three-nucleon forces within the employed framework. The sizes of the required threenucleon force contributions agree well with expectations based on Weinberg's power counting. We identify the energy range in elastic $N d$ scattering best suited to study three-nucleon force effects and estimate the achievable accuracy of theoretical predictions for various observables.

This new semi-local improved chiral nucleon-nucleon interaction was used for the first time to study several electromagnetic and weak processes at energies below the pion production threshold in Ref. [40]. Cross sections and selected polarization observables for deuteron photodisintegration, nucleon-deuteron radiative capture, threebody ${ }^{3} \mathrm{He}$ photo-disintegration as well as capture rates for decays of the muonic ${ }^{2} \mathrm{H}$ and ${ }^{3} \mathrm{He}$ atoms were calculated. The Lippmann-Schwinger and Faddeev equations in momentum space are solved to obtain nuclear states. The electromagnetic current operator is taken as a single nucleon current supplemented by many-body contributions induced via the Siegert theorem. For muon capture processes the non-relativistic weak current together with the dominant relativistic corrections is used. Our results compare well with experimental data, demonstrating the same
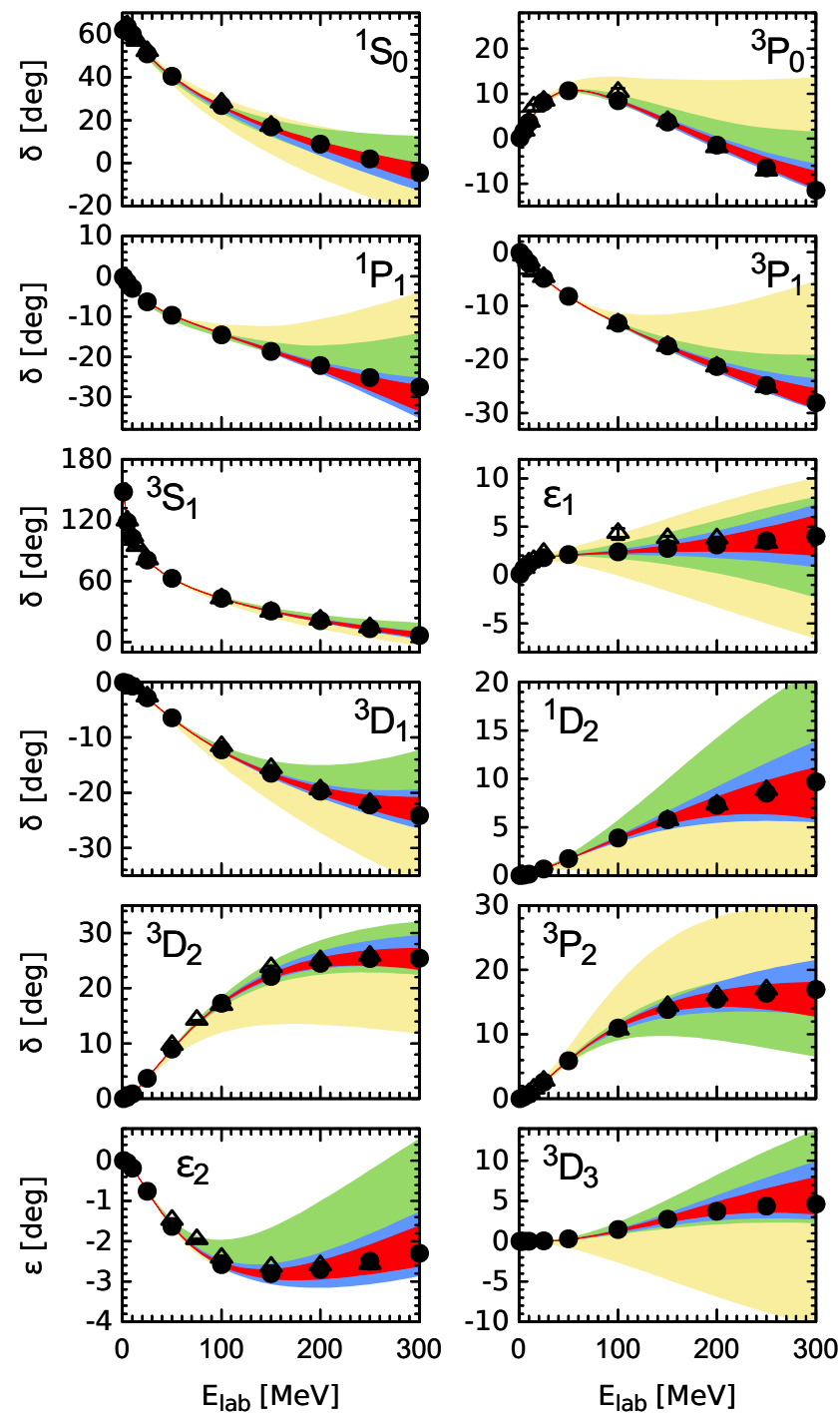

Figure 6. Results for the np S-, $\mathrm{P}$ - and $\mathrm{D}$ - waves and the mixing angles $\epsilon_{1}, \epsilon_{2}$ up to $\mathrm{N}^{4} \mathrm{LO}$ based on the cutoff of $R=0.9 \mathrm{fm}$ in comparison with the Nijmegen PWA [37] (solid dots) and the GWU single-energy PWA [38] (open triangles). The bands of increasing width show estimated theoretical uncertainty at $\mathrm{N}^{4} \mathrm{LO}$ (red), $\mathrm{N}^{3} \mathrm{LO}$ (blue), $\mathrm{N}^{2} \mathrm{LO}$ (green) and NLO (yellow).

quality as is observed for the semi-phenomenological AV18 potential. Compared to the older version of the chiral potential with a nonlocal regularization, a much smaller cut-off dependence is found for the state-of-art chiral local interaction employed in this paper. Finally, estimates of errors due to the truncation of the chiral expansion are given.

\section{Axial currents from chiral EFT}

Few-nucleon electroweak reactions constitute an exciting frontier for nuclear physics and astrophysics. Being an excellent testing ground for chiral EFT, they are intimately related through the same LO short-range operator to such different processes as three-nucleon interactions, P-wave pion production in NN collisions as well as pion photoproduction and radiative capture reactions in the $2 \mathrm{~N}$ system. 
A precise determination of the strength of this operator is the main task of the ongoing MuSun experiment at PSI [41] which will measure the rate of muon capture on the deuteron with a precision of $1.5 \%$. This will allow one to improve the accuracy and reliability of theoretical predictions for nuclear reactions involving neutrinos such as e.g. the solar proton-proton fusion and the hep processes which figure importantly in astrophysics.

The NN contributions to the axial charge and current operators have been first considered in chiral EFT by Park et al. [42, 43] who have, however, omitted certain contributions and ignored pion-pole terms. Recently, the exchange axial currents have been re-considered by Baroni et al. [44] in the framework of time-ordered perturbation theory. These authors have, however, ignored non-static corrections to the NN operators and the contributions of reducible-like three-nucleon diagrams. In Ref. [45], we have performed the complete derivation of the one-, twoand three-nucleon axial charge and current operators up to fourth order relative to the leading one-body contribution using the method of unitary transformation, see section 3 for more details. Notice that the same approach has been used to derive the nuclear forces developed in our group, which are, therefore, by construction consistent with the current operators. Using the freedom to employ additional unitary transformations, we have succeeded to remove all ultraviolet divergences generated by the loop diagrams and to bring the pion-pole contributions into the form which matches exactly the corresponding expressions for the nuclear forces. We expect this feature to be particularly useful for maintaining consistency between regularized expressions for the nuclear forces and current operators. Interestingly, we obtain different results from those of Baroni et al. [44] even for the long-range part of the NN current operator in the static limit. It will be very interesting to apply the resulting expressions to explicitly check consistency between the short-range axial currents and the three-nucleon forces and to study weak few-nucleon processes such as the triton- $\beta$ decay and muon capture reactions on ${ }^{2} \mathrm{H}$ and ${ }^{3} \mathrm{He}$.

\section{Pion production in NN collisions}

The reaction $N N \rightarrow N N \pi$ offers a challenging testing ground for chiral EFT at intermediate energies, see Ref. [46] for a review, and offers important insights into symmetry-breaking pattern of hadronic reactions. In particular, the charge-symmetry-breaking (CSB) process $p n \rightarrow d \pi^{0}$ probes the small but calculable CSB amplitudes which, to leading order in chiral EFT, are proportional to the strong-interaction and electromagnetic contributions to the nucleon mass difference $\delta m_{N}^{\mathrm{str}}$ and $\delta m_{N}^{\mathrm{em}}$, respectively. The strong-interaction mass shift extracted at $\mathrm{LO}$ from the TRIUMF data [47] of the forward-backward asymmetry for the reaction $p n \rightarrow d \pi^{0}, \delta m_{N}^{\text {str, LO }}=1.5 \pm 0.8_{\text {exp }} \pm 0.5_{\text {th }}$ [48], is in a good agreement with the available empirical information on this quantity. However, given the large momenta involved in the pion production reactions, it is important to analyze in detail the convergence of the chiral

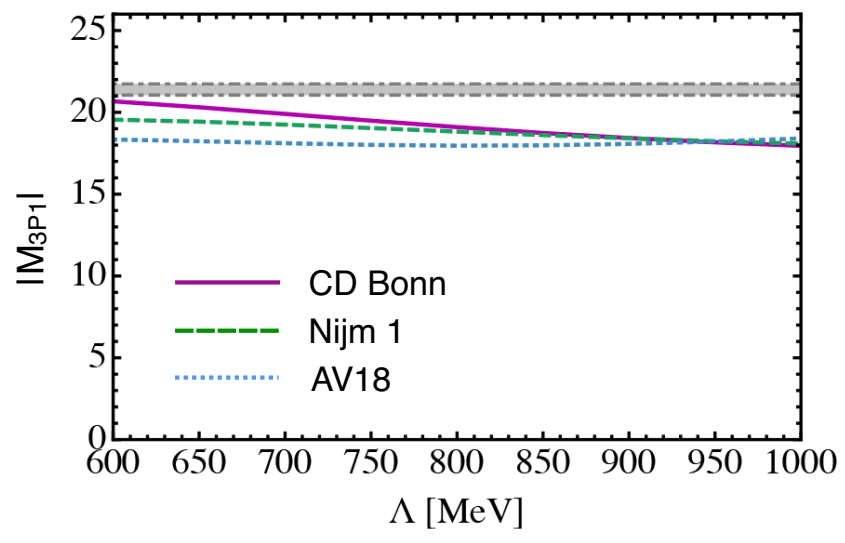

Figure 7. The $p p \rightarrow d \pi^{+}$amplitude $\left|M_{3 P 1}\right|$ at threshold as a function of the cutoff $\Lambda$ imposed in the convolution integrals with various phenomenological NN wave functions. The calculated amplitude includes the complete set of LO, NLO and $\mathrm{N}^{2} \mathrm{LO}$ operators except for the $\mathrm{N}^{2} \mathrm{LO}$ five-point contact term. The horizontal grey band between the dot-dashed lines shows the experimental value of the amplitude.

EFT for such processes. This provides a strong motivation for our $\mathrm{N}^{2} \mathrm{LO}$ study of the near-threshold cross section for the isospin-conserving channel $p p \rightarrow d \pi^{+}$, which is precisely measured in hadronic atom experiments. The various contributions to the corresponding pion production operator up to $\mathrm{N}^{2} \mathrm{LO}$ have already been worked out in Refs. $[49,50]$ where, however, no convolutions with the nuclear wave functions have been performed. Notice that the power counting for pion production reactions differs from the standard one in chiral EFT due to the need to explicitly account for the corresponding momentum scale $p \sim \sqrt{M_{\pi} m_{N}} \simeq 340 \mathrm{MeV}$. The modified counting is referred to as the momentum counting scheme (MCS). Further, given $m_{\Delta}-m_{N} \sim \sqrt{M_{\pi} m_{N}}$, one expects it to be advantageous to treat the $\Delta$-isobar as an explicit degree of freedom.

In Ref. [51], we have analyzed in detail the convergence of the MCS for the reaction $p p \rightarrow d \pi^{+}$. At threshold, only S-wave pion production has to be considered, and the only contribution to the amplitude emerges from the initial $p p^{3} \mathrm{P}_{1}$ partial wave. We found it to be advantageous to employ phenomenological $\mathrm{NN}$ wave functions since the available chiral potentials tend to remove a part of the intermediate-range physics relevant for this reaction. ${ }^{1}$ The amplitude $M_{3 P 1}$ is dominated by the longestrange Weinberg-Tomozawa operator at LO. The NLO corrections are known to undergo significant cancellations and yield a small contribution comparable in size with the $\mathrm{N}^{2} \mathrm{LO}$ correction. We found that the $\mathrm{N}^{2} \mathrm{LO}$ short-range term needed to reproduce the experimental value of the amplitude of $\left|M_{3 P 1}\right|^{\text {exp }}=21.5_{-0.5}^{+0.2}$, extracted from the total cross section in the $n n \rightarrow d \pi^{-}$channel measured at PSI [52], agrees well with the expected size of $\mathrm{N}^{2} \mathrm{LO}$ terms in the MCS, see Fig. 7. Also the dependence of the $\mathrm{N}^{2} \mathrm{LO}$ contribution on the LECs $c_{i}$ appears to be consistent with the estimated uncertainty at this order. Finally, we have

\footnotetext{
${ }^{1}$ This effect is less pronounced for the new generation of chiral potentials of Ref. [32, 36] for the two hardest values of the regulator.
} 
studied the role of the $\Delta$-isobar using different strategies to account for its contributions. We found that the main effects of the $\Delta$-isobar for the considered observable are fairly well represented via saturation of the LECs $c_{i}$. The coupled-channel effects turn out not to exceed the magnitude of $\mathrm{N}^{2} \mathrm{LO}$ effects.

We thank our collaborators Vadim Baru, Arseniy Filin, Jacek Golak, Johann Haidenbauer, Christoph Hanhart, Hiroyuki Kamada, Stefan Kölling, Sebastian König, Hermann Krebs, Simon Kreuzer, Alexander Kudryatsev, Dean Lee, Mark Lenkewitz, Fred Myhrer, Andreas Nogga, Daniel Phillips, Giullermo Rios, Dagmara Rozpędzik, Roman Skibiński, Henryk Witała and all members of the LENPIC Collaboration. The work reported here would not have been possible without the support from the Deutsche Forschungsgemeinschaft within the SFB/TR16.

\section{References}

[1] V. Bernard, N. Kaiser and U.-G. Meißner, Z. Phys. C 70 (1996) 483 [arXiv:hep-ph/9411287].

[2] V. Bernard, N. Kaiser and U.-G. Meißner, Eur. Phys. J. A 11 (2001) 209 [arXiv:hep-ph/0102066].

[3] S. R. Beane, V. Bernard, T.-S. H. Lee, U.-G. Meißner and U. van Kolck, Nucl. Phys. A 618 (1997) 381 [arXiv:hep-ph/9702226].

[4] H. Krebs, V. Bernard and U.-G. Meißner, Eur. Phys. J. A 22 (2004) 503 [arXiv:nucl-th/0405006].

[5] E. Epelbaum, H.-W. Hammer and U.-G. Meißner, Rev. Mod. Phys. 81 (2009) 1773 [arXiv:0811.1338 [nuclth]].

[6] M. Lenkewitz, E. Epelbaum, H.-W. Hammer and U.-G. Meißner, Phys. Lett. B 700 (2011) 365 [arXiv:1103.3400 [nucl-th]].

[7] P. Argan et al., Phys. Lett. 206B (1988) 4 [Erratumibid. B 213 (1988) 564].

[8] M. Lenkewitz, E. Epelbaum, H. W. Hammer and U.-G. Meißner, Eur. Phys. J. A 49 (2013) 20 [arXiv:1209.2661 [nucl-th]].

[9] V. Bernard, B. Kubis and U.-G. Meißner, Eur. Phys. J. A 25 (2005) 419 [nucl-th/0506023].

[10] S. Kölling, E. Epelbaum, H. Krebs and U.G. Meißner, Phys. Rev. C 80, 045502 (2009) [arXiv:0907.3437 [nucl-th]].

[11] E. Epelbaum, W. Glöckle and U.-G. Meißner, Nucl. Phys. A 637, 107 (1998) [arXiv:nucl-th/9801064].

[12] E. Epelbaum, W. Glöckle and U.-G. Meißner, Nucl. Phys. A 671, 295 (2000) [arXiv:nucl-th/9910064].

[13] E. Epelbaum, U.-G. Meißner and W. Glöckle, Nucl. Phys. A 714, 535 (2003) [arXiv:nucl-th/0207089].

[14] E. Epelbaum, Phys. Lett. B 639, 456 (2006).

[15] E. Epelbaum, Eur. Phys. J. A 34, 197 (2007) [arXiv:0710.4250 [nucl-th]].

[16] S. Pastore, R. Schiavilla and J. L. Goity, Phys. Rev. C 78, 064002 (2008) [arXiv:0810.1941 [nucl-th]].

[17] S. Pastore, L. Girlanda, R. Schiavilla, M. Viviani and R. B. Wiringa, Phys. Rev. C 80, 034004 (2009) [arXiv:0906.1800 [nucl-th]].
[18] S. Kölling, E. Epelbaum, H. Krebs and U.G. Meißner, Phys. Rev. C 84 (2011) 054008 [arXiv:1107.0602 [nucl-th]].

[19] S. Pastore, L. Girlanda, R. Schiavilla and M. Viviani, Phys. Rev. C 84 (2011) 024001 [arXiv:1106.4539 [nucl-th]].

[20] D. Rozpędzik, J. Golak, S. Kölling, E. Epelbaum, R. Skibiński, H. Witała and H. Krebs, Phys. Rev. C 83 (2011) 064004 [arXiv:1103.4062 [nucl-th]].

[21] S. Kölling, E. Epelbaum and D. R. Phillips, Phys. Rev. C 86, 047001 (2012) [arXiv:1209.0837 [nucl-th]].

[22] H. Krebs, E. Epelbaum and U.-G. Meißner, Phys. Rev. C 80 (2009) 028201 [arXiv:0812.0132 [hep-th]].

[23] V. Pascalutsa, Phys. Lett. B 503, 85 (2001) [arXiv:hep-ph/0008026].

[24] H. Krebs, E. Epelbaum and U.-G. Meißner, Phys. Lett. B 683 (2010) 222 [arXiv:0905.2744 [hep-th]].

[25] S. Koenig and H.-W. Hammer, Phys. Rev. C 83 (2011) 064001 [arXiv:1101.5939 [nucl-th]].

[26] H.-W. Hammer and D. R. Phillips, Nucl. Phys. A 865 (2011) 17 [arXiv:1103.1087 [nucl-th]].

[27] S. Kreuzer and H.-W. Hammer, Phys. Lett. B 673 (2009) 260 [arXiv:0811.0159 [nucl-th]].

[28] S. Kreuzer and H.-W. Hammer, Eur. Phys. J. A 43 (2010) 229 [arXiv:0910.2191 [nucl-th]].

[29] S. Kreuzer and H.-W. Hammer, Phys. Lett. B 694 (2011) 424 [arXiv:1008.4499 [hep-lat]].

[30] U.-G. Meißner, G. Rios and A. Rusetsky, Phys. Rev. Lett. 114 (2015) 091602 [arXiv:1412.4969 [hep-lat]].

[31] E. Epelbaum, H. Krebs, D. Lee and U.-G. Meißner, Eur. Phys. J. A 41, 125 (2009) [arXiv:0903.1666 [nuclth]].

[32] E. Epelbaum, H. Krebs and U.-G. Meißner, Eur. Phys. J. A 51 (2015) 53 [arXiv:1412.0142 [nucl-th]].

[33] E. Epelbaum, W. Glöckle and U.-G. Meißner, Nucl. Phys. A 747, 362 (2005) [nucl-th/0405048].

[34] D. R. Entem and R. Machleidt, Phys. Rev. C 68, 041001 (2003) [nucl-th/0304018].

[35] W. P. Abfalterer, F. B. Bateman, F. S. Dietrich, R. W. Finlay, R. C. Haight and G. L. Morgan, Phys. Rev. C 63, 044608 (2001).

[36] E. Epelbaum, H. Krebs and U.-G. Meißner, Phys. Rev. Lett. 115 (2015) 122301 [arXiv:1412.4623 [nuclth]].

[37] V. G. J. Stoks et al., Phys. Rev. C 48, 792 (1993).

[38] R. A. Arndt, I. I. Strakovsky and R. L. Workman, Phys. Rev. C 50, 2731 (1994).

[39] S. Binder et al. [LENPIC Collaboration], Phys. Rev. C 93 (2016) 044002 [arXiv:1505.07218 [nucl-th]].

[40] R. Skibinski et al., Phys. Rev. C 93 (2016) 064002 [arXiv:1604.03395 [nucl-th]].

[41] V. A. Andreev et al. [MuCap Collaboration], Phys. Rev. Lett. 99, 032002 (2007) [arXiv:0704.2072 [nuclex]].

[42] T. S. Park, D. P. Min and M. Rho, Phys. Rept. 233, 341 (1993) [hep-ph/9301295]. 
[43] T. S. Park et al., Phys. Rev. C 67, 055206 (2003) [nucl-th/0208055].

[44] A. Baroni, L. Girlanda, S. Pastore, R. Schiavilla and M. Viviani, Phys. Rev. C 93, no. 1, 015501 (2016) Erratum: [Phys. Rev. C 93, no. 4, 049902 (2016)] [arXiv:1509.07039 [nucl-th]].

[45] H. Krebs, E. Epelbaum and U.-G. Meißner, "Nuclear axial current operators to fourth order in chiral effective field theory," arXiv:1610.03569 [nucl-th].

[46] V. Baru, C. Hanhart and F. Myhrer, Int. J. Mod. Phys. E 23, no. 4, 1430004 (2014) [arXiv:1310.3505 [nuclth]].

[47] A. K. Opper et al., Phys. Rev. Lett. 91, 212302 (2003) [nucl-ex/0306027].
[48] A. Filin, V. Baru, E. Epelbaum, J. Haidenbauer, C. Hanhart, A. E. Kudryavtsev and U.-G. Meißner, Phys. Lett. B 681, 423 (2009) [arXiv:0907.4671 [nuclth]].

[49] A. A. Filin, V. Baru, E. Epelbaum, H. Krebs, C. Hanhart, A. E. Kudryavtsev and F. Myhrer, Phys. Rev. C 85, 054001 (2012) [arXiv:1201.4331 [nucl-th]].

[50] A. A. Filin, V. Baru, E. Epelbaum, H. Krebs, C. Hanhart and F. Myhrer, Phys. Rev. C 88, no. 6, 064003 (2013) [arXiv:1307.6187 [nucl-th]].

[51] V. Baru, E. Epelbaum, A. A. Filin, C. Hanhart, H. Krebs and F. Myhrer, Eur. Phys. J. A 52, no. 5, 146 (2016) [arXiv:1602.07333 [nucl-th]].

[52] T. Strauch et al., Phys. Rev. Lett. 104, 142503 (2010) [arXiv:1003.4153 [nucl-ex]]. 